\title{
Análise das variáveis hemodinâmicas no posicionamento gravitacional
}

\section{Analysis of hemodynamic variables on gravitational positioning}

\author{
Fernanda Silva de Sousa ${ }^{[a]}$, José Alexandre Bachur ${ }^{[b]}$, Lígia Melo de Paula ${ }^{[c]}$, Cynthia Kallás Bachur ${ }^{[\mathrm{d}]}$ \\ [a] Graduanda do curso de Fisioterapia da Universidade de Franca (Unifran), Franca, SP - Brasil, e-mail: \\ fers.sousa@hotmail.com \\ [b] Fisioterapeuta, doutor em Patologia, Universidade de Franca (Unifran), Franca, SP - Brasil, e-mail: jabachur@unifran.br \\ [c] Fisioterapeuta, especialista em Reeducação Postural Global, Acupuntura e Pilates Terapêutico, Universidade de Franca \\ (Unifran), Franca, SP - Brasil, e-mail: ligiampfisio@hotmail.com \\ [d] Fisioterapeuta, mestre em Promoção de Saúde, Universidade de Franca (Unifran), Franca, SP - Brasil, e-mail: \\ kabachur@unifran.br
}

\section{Resumo}

Introdução: O sistema nervoso autônomo governa, a curto e médio prazo, as modificações necessárias para adaptação da pressão arterial e da frequência cardíaca às mudanças posturais. Objetivo: Analisar as variáveis hemodinâmicas em indivíduos saudáveis quando submetidos ao posicionamento gravitacional. Materiais e métodos: Sessenta indivíduos saudáveis, divididos por faixa etária em três grupos: GI ( $\mathrm{n}=20)$, de 15 a 20 anos, GII ( $\mathrm{n}=20)$, de 21 a 40 anos, GIII $(\mathrm{n}=20)$, de 41 a 60 anos. Todos foram submetidos ao posicionamento gravitacional e tiveram tanto a frequência cardíaca quanto a pressão arterial monitoradas durante todo o tempo de inversão. Resultados: Houve diminuição significativa da frequência cardíaca e da pressão arterial sistólica após 3, 6, 9 e 12 minutos de inversão quando comparado aos valores de repouso, porém 3 minutos após o retorno à posição ortostática, essas variáveis retornaram aos valores basais. Em relação à pressão arterial diastólica, não foi verificada diminuição significativa no GI e no GII 3 minutos após a inversão, mas no GIII houve queda significativa. Após 6 minutos de inversão somente o GI apresentou queda significativa. Analisando 9 minutos após o posicionamento, somente o GIII apresentou queda significativa. Após 12 minutos de inversão, houve queda da pressão arterial diastólica somente no GIII quando comparado com os valores em repouso. Retornando à posição vertical, todos os grupos retornaram aos valores 
basais. Conclusão: 0 posicionamento gravitacional altera as variáveis hemodinâmicas, causando queda nos valores da frequência cardíaca e pressão arterial durante o período de inversão.

Palavras-chave: Frequência cardíaca. Pressão arterial. Inversão.

\section{Abstract}

Introduction: The autonomic nervous system governs the short and medium term, the necessary modifications to adapt blood pressure and heart rate to changes in posture. Objective: To analyze the hemodynamics variables in healthy individuals when subjected to positional gravity. Materials and methods: Sixty healthy individuals, divided in groups based on age, being GI $(n=20)$, from 15 to 20 years old, GII $(n=20)$, from 21 to 40 years old, GIII $(n=20)$ from 41 to 60 years old, being submitted to the position gravity through the GPS equipment and they had the cardiac frequency and blood pressure monitored throughout all the time of inversion. Results: A significant reduction of heart rate and blood pressure in the rest position when compared after 3, 6, 9 and 12 minutes of inversion, however 3 minutes after return to the orthostatic position this variables came back near to the baseline. About the blood pressure diastolic, it was not observed a significant decrease in the GI and GII group in the rest position when compared after 3 minutes; however in the GIII group it was observed a significant drop. After 6 minutes of inversion only the GI group showed a significant drop. When analyzed 9 minutes after the position, only the GIII group showed a significant dro. After 12 minutes the inversion, it was also showed a blood pressure diastolic drop only in GIII group when compared to the values of the rest position. Returning to the vertical position, all groups returned to the baseline values after 3 minutes. Conclusion: The gravitational positioning changes the hemodynamics variables causing a drop of the values of the heart rate and the blood pressure during the inversion period.

Keywords: Heart rate. Blood pressure. Inversion.

\section{Introdução}

O sistema nervoso autônomo é vital na manutenção da homeostase porque regula a pressão arterial (PA), o balanço hidreletrolítico e a temperatura corporal. É o sistema nervoso autônomo que governa, a curto e médio prazo, as modificações necessárias para adaptação da PA e da frequência cardíaca (FC) às mudanças posturais (1).

Quanto à posição corporal, durante a mudança postural de supino para sentado, ocorrem ajustes cardiovasculares pelo fato de a força da gravidade agir contra o retorno venoso para o coração, resultando num volume sistólico reduzido e FC aumentada para manutenção do débito cardíaco (2).

Ao ficarmos em pé, o retorno venoso para o coração é reduzido, diminuindo a pressão de enchimento, podendo causar um decréscimo de aproximadamente $40 \%$ do volume sistólico (3). Logo que se iniciam as mudanças circulatórias para assumirmos a posição ortostática, ocorre normalmente uma queda na PA e na pressão de enchimento do ventrículo esquerdo. Isso desencadeia um reflexo que estimula receptores de pressão elevada nas carótidas e no arco aórtico, assim como receptores de pressão baixa, localizados no coração e nos pulmões. A queda do retorno venoso pela posição ortostática diminui a pressão nesses receptores, permitindo liberação de seus impulsos, os quais alcançam o tronco cerebral, provocando aumento de carga simpática que resulta em vasoconstrição sistêmica (4).

A gravidade é uma força de compressão que afeta constantemente a estrutura vertical dos seres vivos. Há muito tempo o ser humano vem sentindo os efeitos danosos dessa força, capaz de causar inúmeros malefícios e uma perda significativa da qualidade de vida. Em sua busca incessante pelo conforto absoluto proporcionado pelo grande salto tecnológico ocorrido a partir do século XX, esse homem se tornou excessivamente sedentário e, como consequência disso, passou a sofrer problemas de saúde generalizados, sendo alguns considerados raros até então. No decorrer do século passado, pesquisadores perceberam que a gravidade era uma das maiores causadoras dos desalinhamentos entre as vértebras, e que estes, por mais leves que 
sejam, quando aliados a sedentarismo, problemas genéticos, má postura, ou mesmo acidentes, pressionam as terminações nervosas, gerando inúmeros problemas que se refletem em todo o corpo. Felizmente, esse salto tecnológico permitiu também que se desenvolvessem equipamentos a fim de ajudar o homem em sua busca por mais qualidade de vida, tanto na parte física como mental. Um desses melhoramentos foi proporcionado pelo surgimento de equipamentos que permitem a ele aproveitar a força da gravidade em benefício próprio, ou seja, usá-la para realinhar a estrutura do seu corpo, a própria força que o desalinhou (5).

0 Gravity Power System (GPS) é um equipamento que permite a união da força da gravidade com exercícios respiratórios e posturais, proporcionando então ao indivíduo grandes benefícios ortopédicos, metabólicos, além do desenvolvimento do equilíbrio físico e mental (6).

Porém, ainda não se sabe qual é o comportamento da FC e PA na postura estática de cabeça para baixo (gravitacional), pois não é a ele que o nosso organismo está habituado, podendo então sofrer alterações nessas variáveis.

As inúmeras dúvidas e curiosidades do entendimento das respostas hemodinâmicas no posicionamento gravitacional induziram à realização deste estudo, podendo ser mais uma técnica terapêutica eficaz para o atendimento seguro em várias áreas da fisioterapia. Sendo assim, o objetivo deste trabalho foi analisar as variáveis hemodinâmicas em indivíduos saudáveis em diferentes faixas etárias submetidos ao posicionamento gravitacional.

\section{Materiais e métodos}

Este trabalho foi aprovado pelo Comitê de Ética em Pesquisa da Universidade de Franca, protocolo n. 0011/11. Os participantes concordaram e assinaram o termo de consentimento livre e esclarecido, elaborado conforme a Resolução n. 196/96 do Conselho Nacional de Saúde.

Concordaram em participar deste estudo, alunos e funcionários de uma instituição privada. Foram incluídos aqueles que não fizessem uso de medicação que alterasse os valores da FC e da PA. Os critérios de exclusão para a realização do presente estudo foram: pacientes cardiopatas, gestantes, portadores de doenças neurológicas, indivíduos hemodinamicamente instáveis, pico febril, qualquer tipo de processos infecciosos e/ou alterações hematológicas.

No total, foram estudados voluntariamente 60 indivíduos de ambos os sexos, divididos em grupos por faixa etária: GI $(n=20)$, com idade variando entre 15 e 20 anos; GII $(n=20)$, com idade variando entre 21 e 40 anos; e GIII ( $\mathrm{n}=20)$, com idade variando de 41 a 60 anos, os quais tiveram os parâmetros hemodinâmicos da FC e da PA monitorados nas situações de repouso corporal em posição ortostática pré-inversão (RCO- I), durante o período de posição corporal em inversão ortostática (PCIO) e, em repouso corporal em posição ortostática pós-inversão (RCO-II).

Para verificar a FC, foram acoplados no tórax dos participantes um frequencímetro da marca Polar, tendo como critério de suspensão do posicionamento quando o indivíduo apresentar sintomas e sinais de intolerância à inversão, como medo, vertigem e náuseas.

A aferição da medida indireta da PA se deu utilizando-se esfigmomanômetro aneroide para adultos, previamente calibrado, e um estetoscópio. Essa coleta de dados foi realizada somente por um examinador com o objetivo de se padronizar o modo operacional. A PA dos indivíduos foi monitorada sempre no membro superior direito, e a aferição antes da inversão dos indivíduos foi obtida na posição recomendada na rotina para a medida da pressão arterial: sentada. Para a aferição da PA durante a inversão, solicitou-se aos participantes que posicionassem os braços ao lado do corpo. Como regra geral, recomenda-se que a largura da bolsa inflável corresponda a $40 \%$ da circunferência do membro, de acordo com as orientações estabelecidas na V Diretrizes Brasileiras de Hipertensão Arterial (7).

0 equipamento utilizado permite com que o indivíduo se mantenha preso a uma barra ajustável pelos tornozelos, permitindo que o corpo fique flutuando, liberando as articulações e, principalmente, a coluna vertebral de qualquer impacto ou bloqueio. Antes da aplicação do protocolo sugerido, simulou-se a posição para que o indivíduo se adaptasse ao procedimento (6). Todos eram aconselhados a não se movimentar durante o posicionamento.

Numa planilha específica para este procedimento, foram anotados os valores de FC e PA em repouso, três minutos após a inversão, ficando o indivíduo nesta posição e colhidos tais valores a cada três minutos até completar 12 minutos nesta posição, e retornando à posição vertical, os dados foram novamente colhidos três minutos depois. 
Os dados foram apresentados sob a forma de média e desvio padrão. Os dados referentes às características das amostras foram submetidos ao teste t-Student, com nível de significância estatística aceita de $\mathrm{p}<0,05$.

\section{Resultados}

As características antropométricas dos indivíduos que participaram do estudo estão descritas no Quadro 1.

Conforme os dados do Quadro 2, podemos observar nos três grupos que o valor médio da FC em RCO-I sofreu uma significativa redução mediante o PCIO após três minutos (GI: $p=0,001$, GII: $p=0,003$, GIII: $p=0,009$ ), após seis minutos (GI: $p=0,000$, GII: $\mathrm{p}=0,001$, GIII: $\mathrm{p}=0,002$ ), após nove minutos (GI: $\mathrm{p}=0,001$, GII: $\mathrm{p}=0,001$, GII: $\mathrm{p}=0,003)$, e após 12 minutos de inversão (GI: $p=0,001$, GII: $p=0,001$, GIII: $\mathrm{p}=0,003)$. Contudo, três minutos após o retorno à posição ortostática (RCO-II) em todos os grupos (GI: $p=0,38$, GII: $p=0,72$, GIII: $p=0,30$ ), os valores de FC retornaram próximos aos valores basais, não apresentando diferença estatística em relação ao valor médio de RCO-I.

Da mesma forma podemos observar que em relação ao valor médio da pressão arterial sistólica (PAS) em RCO-I, houve uma significativa redução à medida que os indivíduos foram mantidos ao PCIO após três minutos (GI: $p=0,001$, GII: $p=0,001$, GIII: $p=0,001$ ), após seis minutos (GI: $p=0,001$, GII: $p=0,001$, GIII: $p=$ 0,001 ), após nove minutos (GI: $p=0,001$, GII: $p=0,001$, GIII: $p=0,001$ ) e após 12 minutos de inversão (GI: $p=$ 0,001, GII: $p=0,001$, GIII: $p=0,001$ ). Assim como a FC, a PAS também retornou rapidamente aos seus valores médios de repouso (RCO-I) após três minutos RCO-II, de tal maneira que não foi possível observar distinção estatisticamente significativa em todos os grupos (GI: $p=0,64$, GII: $p=0,62$, GIII: $p=0,78$ ).

Diferentemente do que pode ser observado para os parâmetros de FC e de PAS, em relação aos respectivos valores médios da pressão arterial diastólica (PAD) em RCO-I, enquanto no GI observou-se uma redução significativa de $\mathrm{PCIO}$ apenas aos seis minutos $(p=0,04)$ mantendo-se estatisticamente inalterada nos outros momentos $(\mathrm{p}=0,33$ aos três minutos, $\mathrm{p}=0,20$ aos nove minutos, e $\mathrm{p}=0,06$ aos $12 \mathrm{minu}$ tos), no GII, em nenhum momento houve alterações significativas $(p=0,54$ aos três minutos, $p=0,73$ aos seis minutos, $p=0,22$ aos nove minutos e $p=0,10$ aos $12 \mathrm{~min}$ ). Porém, em GIII, com excessão do observado aos seis minutos ( $\mathrm{p}=0,07)$ de PCIO, houve uma significativa redução da PAD nos outros períodos de aferição ( $p=0,001$ aos três minutos, $p=0,002$ aos nove minutos, e p = 0,002 aos 12 minutos). Assim como a FC e a PAS, os valores médios da PAD dos três grupos ( $p=0,20$ em GI, $p=0,20$ em GII e $p=0,83$ em GIII) em RCO-II mostraram-se estatisticamente semelhantes aos respectivos valores em RCO-I .

Quadro 1 - Valores apresentados em média + desvio padrão

\begin{tabular}{ccccc}
\hline Grupo & Idade (anos) & Peso $(\mathrm{Kg})$ & Altura $(\mathbf{c m})$ & IMC $\left(\mathbf{k g} / \mathrm{cm}^{2}\right)$ \\
\hline I & $17,85 \pm 1,49$ & $56,43 \pm 10,65$ & $1,64 \pm 0,08$ & $21,12 \pm 3,63$ \\
II & $26,65 \pm 5,11$ & $68,61 \pm 17,79$ & $1,69 \pm 0,09$ & $23,80 \pm 4,46$ \\
III & $50,85 \pm 5,34$ & $73,87 \pm 17,09$ & $1,68 \pm 0,10$ & $25,87 \pm 4,05$ \\
\hline
\end{tabular}

Legenda: $\mathrm{Kg}=$ kilograma; $\mathrm{cm}=$ centímetro; IMC = índice de massa corporal

Fonte: Dados da pesquisa.

Quadro 2 - Informações referentes aos valores hemodinâmicos analisados

(Continua)

\begin{tabular}{|c|c|c|c|c|c|c|c|c|c|}
\hline & \multicolumn{3}{|c|}{ FC (bpm) } & \multicolumn{3}{|c|}{ PAS (mmHg) } & \multicolumn{3}{|c|}{ PAD (mmHg) } \\
\hline & G I & G II & G III & G I & G II & G III & G I & G II & G III \\
\hline Repouso & $\begin{array}{l}93,85 \pm \\
17,59\end{array}$ & $\begin{array}{l}88,60 \pm \\
14,86\end{array}$ & $\begin{array}{l}80,25 \pm \\
13,74\end{array}$ & $\begin{array}{l}114,50 \pm \\
8,65\end{array}$ & $\begin{array}{l}114,00 \pm \\
11,14\end{array}$ & $\begin{array}{l}118,00 \pm \\
7,48\end{array}$ & $\begin{array}{l}73,50 \pm \\
9,10\end{array}$ & $\begin{array}{l}73,00 \pm \\
9,00\end{array}$ & $\begin{array}{l}81,00 \pm \\
7,68\end{array}$ \\
\hline
\end{tabular}


Quadro 2 - Informações referentes aos valores hemodinâmicos analisados

(Conclusão)

\begin{tabular}{|c|c|c|c|c|c|c|c|c|c|}
\hline & \multicolumn{3}{|c|}{ FC (bpm) } & \multicolumn{3}{|c|}{ PAS (mmHg) } & \multicolumn{3}{|c|}{ PAD (mmHg) } \\
\hline & G I & G II & G III & G I & G II & G III & G I & G II & G III \\
\hline $3 \min$ & $\begin{array}{l}80,50 \pm \\
16,21^{*}\end{array}$ & $\begin{array}{l}79,75 \pm \\
12,69^{*}\end{array}$ & $\begin{array}{l}72,20 \pm \\
9,11^{*}\end{array}$ & $\begin{array}{l}104,50 \pm \\
14,31^{*}\end{array}$ & $\begin{array}{l}103,00 \pm \\
13,08^{*}\end{array}$ & $\begin{array}{l}107,00 \pm \\
12,69^{*}\end{array}$ & $\begin{array}{l}71,50 \pm \\
11,95\end{array}$ & $\begin{array}{l}71,50 \pm \\
10,14\end{array}$ & $\begin{array}{l}73,50 \pm \\
11,95^{\star}\end{array}$ \\
\hline $6 \mathrm{~min}$ & $\begin{array}{l}78,70 \pm \\
14,12^{*}\end{array}$ & $\begin{array}{l}77,40 \pm \\
11,85^{\star}\end{array}$ & $\begin{array}{l}71,35 \pm \\
8,81^{*}\end{array}$ & $\begin{array}{l}103,00 \pm \\
13,08^{*}\end{array}$ & $\begin{array}{l}101,50 \pm \\
13,52^{*}\end{array}$ & $\begin{array}{l}105,50 \pm \\
10,71^{*}\end{array}$ & $\begin{array}{l}68,50 \pm \\
8,53^{*}\end{array}$ & $\begin{array}{l}72,00 \pm \\
10,77\end{array}$ & $\begin{array}{l}77,00 \pm \\
12,23\end{array}$ \\
\hline $9 \min$ & $\begin{array}{l}79,35 \pm \\
13,15^{\star}\end{array}$ & $\begin{array}{l}76,75 \pm \\
11,80^{*}\end{array}$ & $\begin{array}{l}71,45 \pm \\
7,97^{*}\end{array}$ & $\begin{array}{l}101,00 \pm \\
13,38^{\star}\end{array}$ & $\begin{array}{l}95,50 \pm \\
24,79^{*}\end{array}$ & $\begin{array}{l}104,50 \pm \\
11,17^{\star}\end{array}$ & $\begin{array}{l}69,50 \pm \\
9,21\end{array}$ & $\begin{array}{l}69,00 \pm \\
12,61\end{array}$ & $\begin{array}{l}77,00 \pm \\
9,00^{*}\end{array}$ \\
\hline $12 \min$ & $\begin{array}{l}78,90 \pm \\
13,93^{*}\end{array}$ & $\begin{array}{l}75,20 \pm \\
11,25^{\star}\end{array}$ & $\begin{array}{l}71,00 \pm \\
8,77^{\star}\end{array}$ & $\begin{array}{l}102,00 \pm \\
12,08^{*}\end{array}$ & $\begin{array}{l}100,00 \pm \\
8,94^{*}\end{array}$ & $\begin{array}{l}105,50 \pm \\
11,17^{\star}\end{array}$ & $\begin{array}{l}68,00 \pm \\
9,27\end{array}$ & $\begin{array}{l}69,00 \pm \\
10,44\end{array}$ & $\begin{array}{l}73,00 \pm \\
11,00^{*}\end{array}$ \\
\hline $\begin{array}{c}\text { Após a } \\
\text { ortostase }\end{array}$ & $\begin{array}{l}91,50 \pm \\
16,10\end{array}$ & $\begin{array}{l}87,55 \pm \\
16,88\end{array}$ & $\begin{array}{l}83,45 \pm \\
10,35\end{array}$ & $\begin{array}{l}115,50 \pm \\
10,23\end{array}$ & $\begin{array}{l}115,00 \pm \\
8,66\end{array}$ & $\begin{array}{l}117,50 \pm \\
8,87\end{array}$ & $\begin{array}{l}77,00 \pm \\
9,00\end{array}$ & $\begin{array}{l}75,50 \pm \\
9,21\end{array}$ & $\begin{array}{l}81,50 \pm \\
8,53\end{array}$ \\
\hline
\end{tabular}

Legenda: FC = frequência cardíaca; PAS = pressão arterial sistólica; PAD = pressão arterial diastólica; bpm = batimentos por minuto; $\mathrm{mmHg}=$ milímetros de mercúrio; $p$ = nível de significância.

Fonte: Dados da pesquisa.

\section{Discussão}

Alguns comentários em relação aos materiais e métodos devem ser esclarecidos antes de iniciarmos a discussão dos dados obtidos. A primeira observação propriamente dita diz respeito à medida da PA pelo método auscultatório. Segundo Leite e Farinatti (8), uma das críticas que surgem é a possibilidade de se ter subestimado os valores de pressão, considerando a precisão de métodos invasivos como o cateterismo intra-arterial. É certo que valores absolutos de PA fornecidos por técnicas invasivas possuem maior validade e confiabilidade. No entanto, essa avaliação foi realizada em indivíduos saudáveis - sendo o cateterismo um procedimento invasivo, reveste-se de riscos consideráveis (dor, espasmo arterial, trombose, estenose, síncope vaso-vagal, hemorragia etc.). Com esse procedimento invasivo, considerado padrão ouro (9), promover riscos à sua aplicação extrapolaria os limites éticos da investigação científica (10). Alguns autores (11) questionam a aplicabilidade dos métodos invasivos em quantificar a pressão sistêmica, uma vez que são extremamente sensíveis aos pulsos de pressões durante a atividade, e menos à detecção do fluxo (9).

É reconhecido que a medida pelo método auscultatório tende a subestimar os valores absolutos da PA; entretanto, em exercícios mais intensos, comparando-se valores obtidos pelo método auscultatório, a relação entre eles tende a ser mantida, conforme o descrito em alguns estudos (12). Considerando o viés de que a subestimação da PA ocorre de forma sistêmica, a associação de uma atividade com maiores ou menores valores de PA pode ser detectada. Portanto, em razão da natureza da solicitação do posicionamento proposto neste estudo, isso parece ser o suficiente.

A metodologia aplicada no presente estudo demonstrou-se segura e de fácil aplicabilidade. Embora não haja trabalhos que estudem os aspectos das variáveis hemodinâmicas na posição gravitacional, os resultados apresentados aqui mostraram uma queda significativa tanto na FC quanto na PA em todas as faixas etárias.

A FC de repouso é influenciada por diversos fatores, como características genéticas, antropométricas, idade, gênero, fatores hormonais, emocionais, nível de aptidão física, estado de saúde, dentre outros (13), e suas influências podem ser analisadas a partir de testes posturais $(14,15)$.

Segundo alguns autores $(16,17)$, os ajustes da modulação da FC da postura supina para sentada são decorrentes dos desvios hidrostáticos causados pelo deslocamento do sangue da região central para as regiões inferiores, reduzindo o débito cardíaco, a pressão arterial e a ativação dos receptores arteriais e cardiopulmonares.

Em condição de repouso, temos interação simpatovagal, porém o predomínio de um em relação ao outro pode variar de acordo com a mudança postural. Dessa forma, um estudo da variabilidade da FC em 
repouso nas posturas supina e sentada nos permite identificar alterações do balanço simpatovagal sobre o nó sinusal (16). Entende-se que, em supino, ocorre maior ativação parassimpática sobre o nó sinusal, em relação à posição sentada (18). Zuttinet al. (19) observaram diminuição da atuação simpática em relação à parassimpática na postura supina, apresentando maior variabilidade da FC.

Da mesma maneira, no trabalho de Jesus (20), que estudou homens e mulheres em uma faixa etária bastante ampla (de 19 a 45 anos), foram encontradas diferenças significativas na transição de supino para a posição ortostática. Em decorrência do envelhecimento, ocorrem alterações do sistema cardiovascular, com mudanças estruturais e funcionais na sensibilidade dos tecidos, no sistema de condução cardíaca, nos vasos sanguíneos e barorreceptores, aumento da rigidez miocárdica e diminuição no enchimento ventricular, que refletem na hemodinâmica (21). Além disso, ocorrem alterações na modulação do sistema nervoso autônomo no coração (22).

A definição de hipotensão ortostática de acordo com The Joint Consensus Committee of the American Autonomic Society e The American Academy of Neurology, de 1996, é uma queda na PAS maior ou igual a $20 \mathrm{mmHg}$ e/ou uma queda na PAD maior ou igual a $10 \mathrm{mmHg}$, no momento em que um indivíduo se move da posição supina (deitado) para a posição ortostática (em pé) ou dentro de três minutos após a ortostase (23). A hipotensão ortostática pode ser sintomática ou assintomática e esses sintomas desaparecem ao passar de um minuto e variam de intensidade de acordo com o indivíduo (24). Jansen et al. (25) observaram que alguns indivíduos têm o declínio imediato da pressão ao ficarem em pé, enquanto outros apresentam um declínio gradual em três minutos ou mais na posição ortostática. Já Seligraet al. (26) registram um maior número de indivíduos com queda na pressão já no primeiro minuto $(73,7 \%$ dos casos).

A ioga, considerado por alguns autores como um tipo de ginástica ou atividade física, é uma antiga filosofia oriental que busca unir as funções psíquicas e físico-orgânicas do ser humano, integrando os sistemas fisiológicos e a mente (27). Desde o seu surgimento, por volta de cinco mil anos, a ioga já considerava a postura estática de cabeça para baixo (posturas invertidas) como a mais benéfica de toda uma série de exercícios criados para manter o corpo do praticante sempre no eixo, alongando e tonificando (6). Essas posições invertidas oferecem um benefício não encontrado em nenhum outro tipo de prática desportiva. Quando o indivíduo está nessa posição, o sangue corre mais facilmente para a cabeça, alimentando os neurônios e ativando todas as funções cerebrais: raciocínio, memória, concentração, criatividade etc. A circulação nas pernas diminui, evitando varizes e o cansaço tão comum nessa área. Além disso, ocorre a inversão da ação da força de gravidade, que constantemente puxa para baixo nossos tecidos e órgãos.

Quando executadas, as posições invertidas reposicionam os órgãos e dão um descanso aos músculos que os sustentam. Além disso, a hipófise - a principal glândula do sistema endócrino, responsável por coordenar a função de todas as outras - é ativada. Vijayalakshmi et al. (28) estudaram os efeitos anti-hipertensivos da ioga em 13 pacientes com hipertensão essencial, que, após terem praticado sessões de 60 minutos diários, seis dias por semana durante um mês, apresentaram reduções significantes na pressão arterial sistólica, na pressão arterial diastólica e na frequência cardíaca. Murugesan et al. (29) observaram 33 pacientes hipertensos em um período de intervenção maior, 11 semanas, e também perceberam melhora no controle da PA. Neste estudo, as quedas das variáveis analisadas mantiveram-se dentro dos valores de normalidade, não causando sintomas de baixo débito cardíaco.

\section{Conclusão}

Com base nos resultados deste trabalho, podemos sugerir que o posicionamento gravitacional causa queda nos valores de FC e PA durante o período de inversão e retornam aos valores basais três minutos após o retorno à posição ortostática. As quedas dessas variáveis se mantiveram dentro dos valores de normalidade, não causando sintomas de baixo débito cardíaco. Contudo, sugerimos novos estudos para aprimorar esses resultados na aplicabilidade em indivíduos com diferentes patologias, podendo ser mais um recurso seguro e eficaz na terapêutica. Os resultados demonstram que essa técnica pode ser mais um recurso seguro e eficaz para procedimentos fisioterapêuticos.

\section{Agradecimentos}

À proprietária do consultório fisioterapêutico, Dra. Lígia Melo de Paula, e ao sr. Odílio Moreira, pela 
valiosa disponibilidade do espaço físico e dos equipamentos utilizados neste estudo, os quais subsidiaram os resultados analisados no presente trabalho.

\section{Referências}

1. Wieling W, Lishout J. Maintennance of postural nomatension in human. In: Low $\mathrm{P}$, editor. Clinical autonomic disards. London: Little Brown; 1993. p. 69-73.

2. Lindqvist A. Noninvasive methods to study autonomic nervous control of circulation. Acta Physiol Scand Suppl. 1990;588:1-107.

3. Grubb BP. Neurocardiogenic syncope. In: Grubb BP, Olshansky B, editors. Syncope: mechanisms and management. Armonk: Futura Publishing; 1998.

4. Bernarroch E. The central autonomic network: funcional organization dysfunction and perspective. Mayo Clin. Proc. 1993;68(10):998-1001.

5. Kosinski D, Grubb BP, Temesy-Armos P. Pathopysiologicalaspects os neurocardiogenicsyncope. Pacing Clin Electrophysiol. 1995;18(4Pt1):716-24. doi:10.1111/j.1540-8159.1995.tb04666.x.

6. Gravity Power System - GPS. [Citado 7 Jan. 2011]. Disponível em: http://www.gravitacional.com.br.

7. Sociedade Brasileira de Hipertensão, Sociedade Brasileira de Cardiologia, Sociedade Brasileira de Nefrologia. V Diretrizes Brasileiras de Hipertensão Arterial. Arq Bras Cardiol. 2006;82(Supl IV).

8. Leite T, Farinatti P. Estudo da freqüência cardíaca, pressão arterial e duplo-produto em exercícios resistidos diversos para grupamentos musculares semelhantes. Rev Bras Fisiol Exerc. 2003;2:68-88.

9. MacDougall JD, McKelvie RS, Moroz DE, Sale DG, McCartney N. Factors affecting blood pressure during heavy weight lifting and static contractions. J Appl Physiol. 1992;73:1590-7. PMid:1447109.

10. Perloff D, Grim C, Flack J, Frohlich E, Hill M, McDonald M. Human blood pressure determination by sphygmomanometry. Circulation. 1993;88:2460-7. doi: 10.1161/01.CIR.88.5.2460

11. Polito MD, Farinatti PTV. Considerações sobre a medida da pressão arterial em exercícios contra-resistência. Rev Bras Med Esporte. 2003;9:25-33. doi:10.1590/S1517-86922003000100005.
12. Simão R, Polito MD, Lemos A. Duplo-produto em exercícios contra-resistidos. Fit Perfor J. 2003;2:279-84. doi:10.3900/fpj.2.5.279.p.

13. Gallo L Jr, Maciel BC, Marin-Neto JA, Martins LEB, Lima EC Filho, Golfetti R. Control of heart rate during exercise in health and disease. Braz J Med Biol Res. 1995;28(11-12):1179-84. PMid:8728845.

14. Marães VRFS, Santos MDB, Catai AM, Moraes FR, Oliveira L, Gallo L Jr, et al. Modulação do sistema nervoso autonômico na resposta da frequência cardíaca em repouso e à manobra de valsalva com o incremento da idade. Rev Bras Fisioter. 2004;8(2):97-103.

15. Sakabe DI, Catai AM, Neves VFC, Oliveira L, Silva de Sá MF, Azevedo GB, et al. Análise da modulação do coração durante condições de repouso em homens de meia-idade e mulheres pós-menopausa. Rev Bras Fisioter. 2004;8(1):89-95.

16. Acharya UR, Kannathal N, Hua LM, Yi LM. Study of heart rate variability signals at sitting and lying postures. J Bodywork MovTheraphies. 2004;2-8. PMid:14992698.

17. Lindquist A. Noninvasive methods to study autonomic nervous control of circulation. Acta Physiol Scand Suppl. 1990;588:1-107.

18. Malik M, Camm AJ. Heart rate variability. Clin Cardiol. 1990;13(8):570-6. doi:10.1002/clc.4960130811.

19. Zuttin RS, Moreno MA, César MC, Martins LEB, Catai AM, Silva E. Avaliação da modulação autonômica da frequência cardíaca nas posturas supina e sentada de homens jovens sedentários. Rev Bras Fisioter. 2008;12(1):7-12. doi:10.1590/ S1413-35552008000100003.

20. Jesus PC. Considerações metodológicas e caracterização de procedimentos implicados nas análises temporal e espectral da variabilidade da freqüência cardíaca, para avaliação clínica do controle autonômico do coração [dissertação]. Brasília: Universidade de Brasília; 1996.

21. Walsh RA. Cardiovascular effects of the aging process. Am J Med. 1987; 82(1B):34-40. doi:10.1016/ 0002-9343(87)90269-5.

22. Lakatta EG. Cardiovascular system. In: Masoro EJ, editor. Handbook of physiology. A critical, comprehensive presentation of physiology knowledge and concepts. New York: Oxford University Press; 1995. 
23. Heitterachi E, Lord SR, Meyerkort P, McCloskey J, Fitzpatrick R. Blood pressure changes on upright tilting predict falls in older people. Age Ageing 2002;31(3):181-6. doi:10.1093/ageing/31.3.181.

24. Consensus statement on the definition of orthostatic hypotension, pure autonomic failure and multiple system atrophy. Neurology. 1996;46:1470. doi:10.1212/ WNL.46.5.1470.

25. Jansen RW, Kelly-Gagnon MM, Lipsitz LA. Intraindividual reproducibility of postprandial and orthostatic blood pressure changes in older nursing-home patients: relationship with chronic use of cardiovascular medications. J Am Geriatr Soc. 1996; 44(4):383-9. PMid:8636581.

26. Seligra LL, Mürrer G, Costa PLN, Grizante P. Hipotensão ortostática em pacientes idosos ambulatoriais, Arq Med ABC. 2007;32(1):17-20.

27. Warrier G, Gunawan G. The complete illustrated guide to Ayurveda. The ancient Indian healing tradition. Shaftesbury: Barnes \& Noble; 1997.
28. Vijayalakshmi P, Madanmohan, Bhavanani AB, Patil A, Babu K. Modulation of stress induced by isometric handgrip test in hypertensive patients following yogic relaxation training. Indian J Physiol Pharmacol. 2004;48(1):59-64. PMid:15270370.

29. Murugesan R, Govindarajulu N, Bera TK. Effect of selected yogic practices on the management of hypertension. Indian J Physiol Pharmacol. 2000;44(2):20710. PMid:10846637.

Recebido: 19/10/2011

Received: 10/19/2011

Aprovado: 03/05/2012

Approved: 05/03/2012 